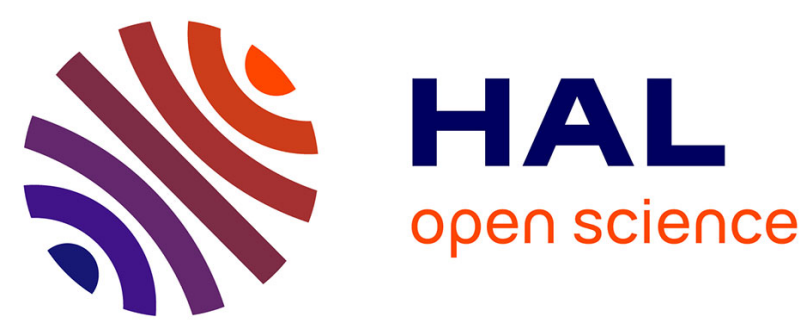

\title{
Electronic interactions between Graphene and cobaltite thin film La0.7Sr0.3CoO3 and its magnetic consequences
}

Zied Othmen, Riadh Othmen, Kais Daoudi, Michel Boudard, Antonella

Cavanna, Ali Madouri, Pascale Gemeiner, Doru C Lupascu, Meherzi Oueslati, Brahim Dkhil

\section{To cite this version:}

Zied Othmen, Riadh Othmen, Kais Daoudi, Michel Boudard, Antonella Cavanna, et al.. Electronic interactions between Graphene and cobaltite thin film La0.7 $\mathrm{Sr} 0.3 \mathrm{CoO} 3$ and its magnetic consequences. Surfaces and Interfaces, 2021, 23, pp.100919. 10.1016/j.surfin.2020.100919 . hal-03369133

\section{HAL Id: hal-03369133 https://hal.science/hal-03369133}

Submitted on 7 Oct 2021

HAL is a multi-disciplinary open access archive for the deposit and dissemination of scientific research documents, whether they are published or not. The documents may come from teaching and research institutions in France or abroad, or from public or private research centers.
L'archive ouverte pluridisciplinaire HAL, est destinée au dépôt et à la diffusion de documents scientifiques de niveau recherche, publiés ou non, émanant des établissements d'enseignement et de recherche français ou étrangers, des laboratoires publics ou privés. 


\title{
Electronic interactions between Graphene and cobaltite thin film $\mathrm{La}_{0.7} \mathrm{Sr}_{0.3} \mathrm{CoO}_{3}$ and its magnetic consequences
}

Zied Othmen ${ }^{1}$, Riadh Othmen ${ }^{2}$, Kais Daoudi ${ }^{3,4}$, Michel Boudard ${ }^{5}$, Antonella Cavanna ${ }^{6}$, Ali Madouri $^{6}$, Pascale Gemeiner ${ }^{1}$, Doru C. Lupascu ${ }^{7}$, Meherzi Oueslati ${ }^{3}$ and Brahim Dkhil ${ }^{1}$

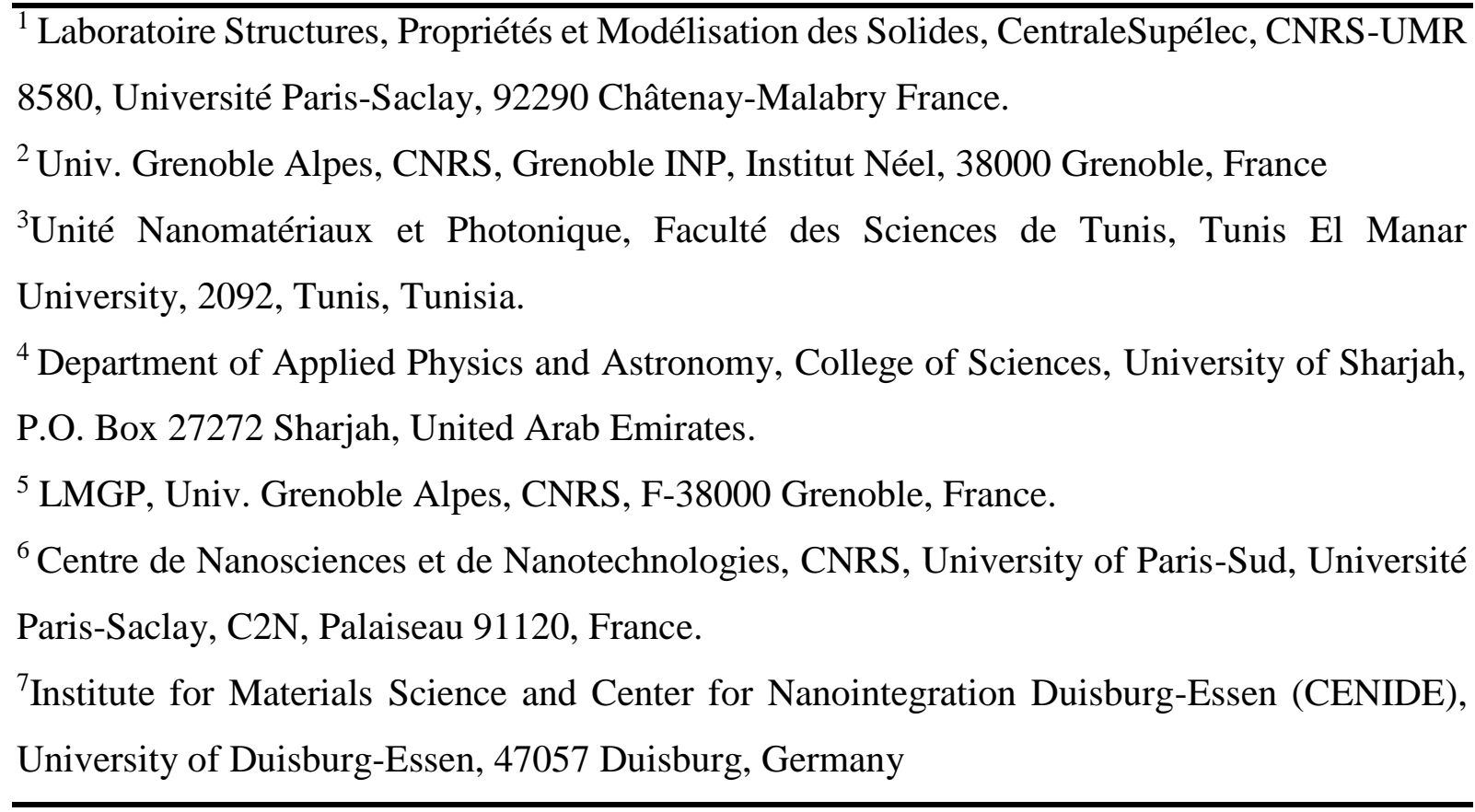

\begin{abstract}
We have successfully synthesized and transferred graphene (Gr) monolayers on top of epitaxial mixed valence $\mathrm{La}_{0.7} \mathrm{Sr}_{0.3} \mathrm{CoO}_{3}$ (LSCO) thin films. Raman spectroscopy reveals that Jahn-Teller (JT) modes associated with the oxygen octahedral distortions usually unobserved for bare LSCO are activated by the deposited graphene. The appearance of these JT modes in the Gr/LSCO heterostructure is attributed to the electronic interactions at the interface between the graphene and the LSCO thin film promoting intermediate spin states of the Co ions. As a result, the magnetic properties of LSCO are affected. Indeed, magnetization measurements show a phase transition at $\sim 135 \mathrm{~K}$ which is due to the presence of the graphene while the ferromagnetic transition of bare LSCO films is observed at $200 \mathrm{~K}$. This magnetic phase is confirmed by Raman spectroscopy measurements as a function of temperature revealing a vibrational transition around the same temperature.
\end{abstract}




\section{Introduction}

Hole or electron doped cobaltite of the type $\mathrm{LaCoO}_{3}$ evokes research interest in the mixed valence transition metal-oxides with perovskite and related structures [1,2]. The introduction of additional charges (holes or electrons), for example, by the substitution of $\mathrm{La}^{3+}$ in $\mathrm{LaCoO}_{3}$ yields remarkable changes in their magnetic and electrical properties $[1,3,4]$. The complex oxide provides many opportunities for the observation of new physical phenomena with promising applications in future electronic devices [5-7]. Due to their interesting magnetic and electrical properties, cobaltites in thin film form are increasingly requested in a wide range of applications, ranging from components in solid oxides fuel cells, oxygen separation membranes and electrochemical reactors, to thermoelectric devices. $\mathrm{La}_{0.7} \mathrm{Sr}_{0.3} \mathrm{CoO}_{3}$ (LSCO), cobaltite is known to be a mixed-valence system $\left(\mathrm{Co}^{3+} / \mathrm{Co}^{4+}\right)$ characterized by rich and complex physical properties [8]. The Co-ions $\left(\mathrm{Co}^{3+}\right)$ and $\left(\mathrm{Co}^{4+}\right)$ can take three different spin states: low-spin (LS), intermediate-spin (IS) and high-spin (HS). The IS states of $\mathrm{Co}^{3+}$ and $\mathrm{Co}^{4+}$ are expected to be Jahn-Teller (JT) active and give rise to $\mathrm{CoO}_{6}$ octahedral distortions [9-12]. The spin states of the Co ions govern the magnetism and the electrical conduction. They are sensitive to external parameters such as temperature, hydrostatic pressure, and doping level [10-12]. It is generally admitted that the ferromagnetism in these mixed-valence systems arises from the double exchange (DE) interaction between $\mathrm{Co}^{3+}$ and $\mathrm{Co}^{4+}$ which also accounts for the electrical conductivity in the ferromagnetic metallic phase $[8,13]$. In our previous studies of epitaxial LSCO films, we have shown that the resistivity is decreased by several orders of magnitude when the thickness is increased from $20 \mathrm{~nm}$ to $100 \mathrm{~nm}$ [14]. In the fully strained $20 \mathrm{~nm}$ thick LSCO film, the conduction mechanism was dominated by $3 \mathrm{D}$ variable range hopping (VRH) over the whole temperature range, and like in the $100 \mathrm{~nm}$ thick film, a ferromagnetic transition occurs around $200 \mathrm{~K}$ like the bulk sample. In this study, a graphene monolayer is transferred on top of the cobaltite thin film in view of introducing an additional perturbation of the electronic structure of the LSCO films. The type of electronic interactions between the LSCO and the graphene are quite interesting and might be of great interest for some electronic applications combining the outstanding physical properties of both materials.

Graphene is a mono atomic thin layer with $\mathrm{sp}^{2}$ bonded carbon atoms arranged in a honeycomb lattice. It has attracted enormous attention thanks to its remarkable electronic properties $[15,16]$. On the other hand, graphene is chemically and physically stable, offering a possibility to combine it with other materials and to create hybrid systems [16,17]. Large area samples of graphene can be obtained by chemical-vapor deposition (CVD) and can 
subsequently be combined with various substrates $[18,19]$ by chemical dissolution and a mechanical transfer method. Generally, the resulting direct contact between graphene layer and the surface of the substrate leads to an exchange of electrons that is realized at the interface between the two materials [19-21]. Combining graphene with semiconducting metal-oxide has been proven as a cost-effective approach for improving fast charge transfer [22]. And in case of cobaltites such as LSCO this approach can therefore be a cheap and efficient way for tuning their electronic and magnetic properties which are controlled by the amount of electron/hole charges. Such graphene-based spin electronic devices possess a remarkable capability fordensity non-volatile memories, reconfigurable electronic devices and, plausibly, solid-state quantum computing devices [20]. The Raman spectrum of graphene contains a $\mathrm{G}$ band associated to the double degenerated zone center $\mathrm{E}_{2 \mathrm{~g}}$ mode, a $2 \mathrm{D}$ band associated to the secondorder zone-boundary phonons and a D band indicating the existence of defects [20,21,23,24]. The frequencies of these peaks are strongly influenced by structural modifications (e.g., doping and stress) and allows tracking any modification of the interactions in the Gr/LSCO system.

In the present work, we investigated the effect of transferring a graphene layer on top of a LSCO thin film (Gr/LSCO) and its consequence on the Co (spin, valence) state and local structure distortions. We unambiguously show changes in the Raman spectrum of Gr/LSCO which drastically differs from that of bare LSCO films. This is due to a charge transfer at the interface between graphene and the LSCO film. The charge transfer affects the magnetic properties of the LSCO film. The study of the temperature dependence of both, the G and the $2 \mathrm{D}$, bands of the graphene layer demonstrates the correlation of their positions with the physical properties of the LSCO film. These results open a new path for tuning physical properties of systems using graphene electrodes combined with mixed valence oxides. For instance, graphene electrodes may serve as a "charge reservoir" in LSCO mixed valence systems and could open new perspectives in hybrid graphene nanostructures with oxides.

\section{Experimental}

LSCO $20 \mathrm{~nm}$-thick films were epitaxially grown on $\mathrm{LaAlO}_{3}$ (LAO) single crystalline substrates using a conventional metal organic deposition (MOD) process. This technique requires an annealing at a relatively high temperature which might be a handicap for further integration in silicon based electronic devices but permits high quality epitaxial film growth. The details of the growth of the LSCO thin films and their structural, microstructural and some physical properties are reported in our previous studies [14,25]. The graphene layers, synthesized on copper foils using a CVD technique and coated with polymethyl methacrylate (PMMA) layers, were transferred 
on top of LSCO films ( $\mathrm{Gr} / \mathrm{LSCO})$ and $\mathrm{SiO}_{2}$ substrates [26-28] $\left(\mathrm{Gr} / \mathrm{SiO}_{2}\right) . \mathrm{Gr} / \mathrm{SiO}_{2}$ is used as a reference sample. Two-dimensional Raman maps of the same area were collected, using the excitation energy $E_{\text {laser }}=2.33 \mathrm{eV}$ of a Renishaw inVia Raman Microscope. A 2400 1/mm grating was used with a spectral resolution of $0.8 \mathrm{~cm}^{-1}$. The temperature was varied from $100 \mathrm{~K}$ to 300 $\mathrm{K}$ with a Linkam THMS600 cell integrated in the Renishaw inVia Raman Microscope. The laser power was fixed to $0.8 \mathrm{~mW}$ to ensure that laser-induced heating does not introduce artefacts. The standard mode laser spot size is around 1.5 microns at $50 \mathrm{x}$ magnification, but we used a smaller spot size of 0.5 microns using confocal mode with a measurement step 0.2 microns for all Raman maps. All peaks were fitted with a single Lorentzian distribution. The magnetic characterization was performed using a commercial SQUID magnetometer (Quantum Design, 5T) with an applied magnetic field of 500 Oe for field cooling (FC) in the $5-400 \mathrm{~K}$ temperature range. Temperature dependence resistivity measurements were performed using a four-terminal technique.

\section{Results and discussion}

Two-dimensional maps of the $\mathrm{G}$ and 2D bands and the corresponding intensity ratios $\left(I_{2 D} / I_{G}\right)$ of $\mathrm{Gr} / \mathrm{SiO}_{2}$ and $\mathrm{Gr} / \mathrm{LSCO} / \mathrm{LAO}$ hybrid structures are shown in Fig.1 (a). Raman mapping of $2 \mathrm{D}$ bands shows that the 2D band positions of graphene deposited on LSCO are shifted to lower frequencies by an amount of $20 \mathrm{~cm}^{-1}$ in comparison to the position of the 2D band of graphene deposited on $\mathrm{SiO}_{2}$. The intensity ratios of the $2 \mathrm{D}$ to the $\mathrm{G}$ bands $\left(I_{2 D} / I_{G}\right)$ are between 1.2 and $4.2 \mathrm{in} \mathrm{Gr} / \mathrm{SiO}_{2}$ and 1.4 and $3.6 \mathrm{in} \mathrm{Gr} / \mathrm{LSCO}$. These features when compared to $\mathrm{Gr} / \mathrm{SiO}_{2}$ reference sample correspond to typical CVD-derived monolayer graphene with defects [23,24,26,28]. The doping of graphene by holes or electrons may therefore be responsible for these shifts [29]. To check the possibility of charge-transfer between the graphene and LSCO film, we follow not only the shift in frequencies of the graphene Raman modes, but also the LSCO Raman modes that are sensitive to changes of the electronic configuration of the Co ions $[9,30,31]$.

The Raman spectrum of the LSCO before the transfer of the graphene is dominated by three main vibrational modes at 200, 423 and $700 \mathrm{~cm}^{-1}$ (Fig. 1 (b)). After the transfer of the graphene monolayer, we note the appearance of four additional modes at 259, 521, 616, 659 and $690 \mathrm{~cm}^{-1}$. The observation of new peaks in such cobaltite material proves an electronic interaction and a change in the electronic configuration of the Co ions [9,30,31], promoting the IS states in $\mathrm{Co}^{3+}$ and/or $\mathrm{Co}^{4+}$, which in turn activate the Raman modes associated to the JT distortions [30]. 
Changes in the magnetic properties by valence state modification were further confirmed by measuring the temperature dependence of the magnetization $\mathrm{M}(\mathrm{T})$, and magnetic hysteresis loops $\mathrm{M}(\mathrm{H})$ before and after the transfer of the graphene layer as shown in Fig. $2(\mathrm{a}, \mathrm{b})$ and $(\mathrm{c})$ respectively. The $\mathrm{M}(\mathrm{T})$ curves of the LSCO film are characterized by a ferromagnetic (FM)like to paramagnetic (PM) transition at a temperature of $T_{C} \sim 200 \mathrm{~K}$.
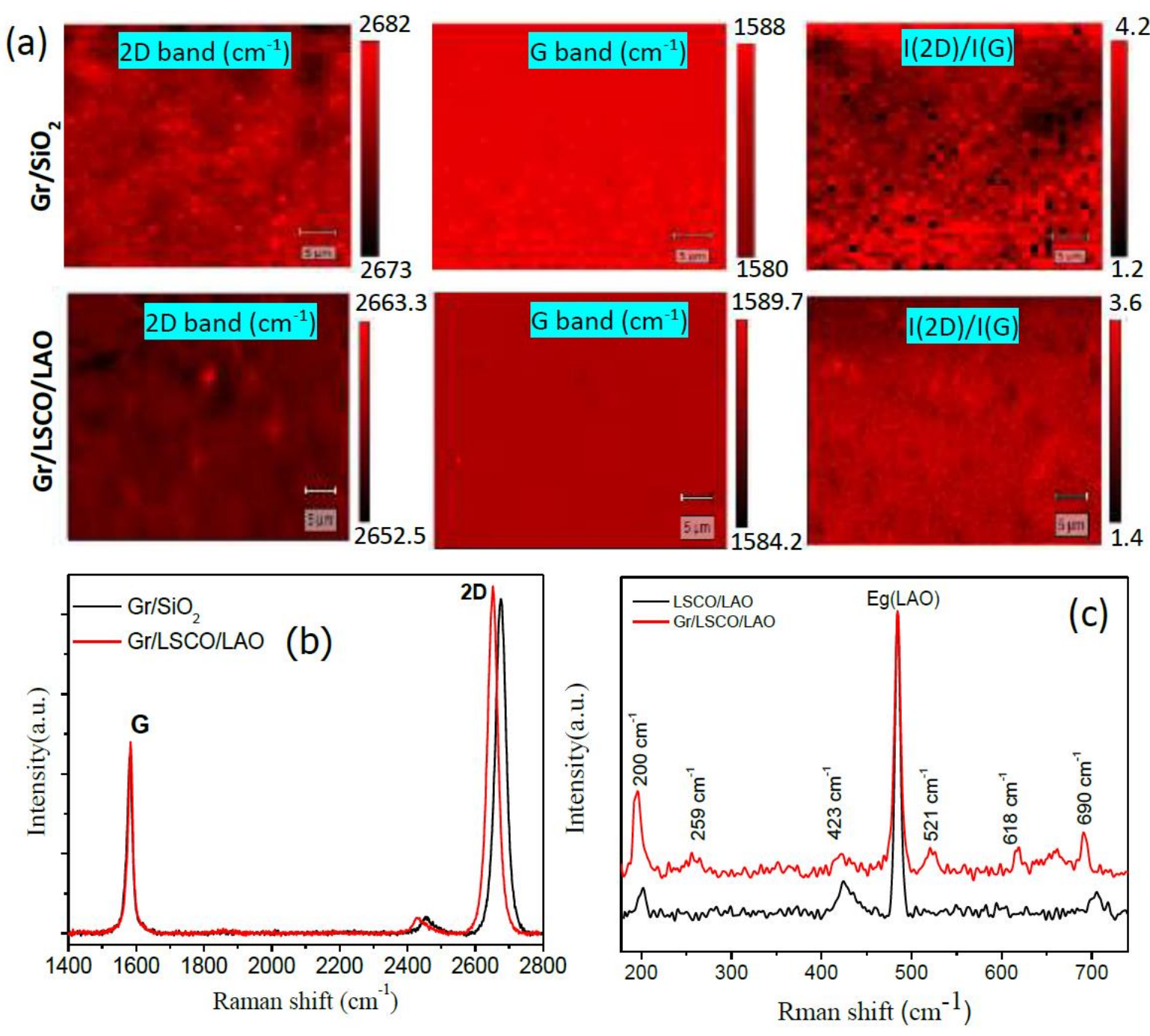

Figure 1: (colour online) (a) First-row and second-row show the two-dimensional Raman spectroscopy maps at room temperature of $\mathrm{G}$ and $2 \mathrm{D}$ modes and the intensity ratios of the $2 \mathrm{D}$ to the $\mathrm{G}$ bands $\left(I_{2 D} / I_{G}\right)$ for $\mathrm{Gr} / \mathrm{SiO} 2$ and $\mathrm{Gr} / \mathrm{LSCO} / \mathrm{LAO}$, respectively. (b) Typical Raman spectra of the graphene transferred on LSCO (red) and on $\mathrm{SiO}_{2}$ (black). (c) Raman spectra at low frequencies of the LSCO (black) and Gr/LSCO (red).

The magnetization significantly drops down when the graphene is deposited onto the LSCO film (Fig. 2 (a)). Such a decrease of the magnetization of the LSCO film may be 
explained by a spin state transition from high-spin (HS) to intermediate-spin (IS) state in $\mathrm{Co}^{+3 /+4}$ due to the charge transfer to graphene. By determining the minimum value of the derivative $d M / d T$ in the temperature dependence of the magnetization (inset Fig. 2 (b)) of the $\mathrm{Gr} / \mathrm{LSCO} / \mathrm{LAO}$, we note the presence of two magnetic phases with two values of critical $T_{C}$ temperature $\left(T_{C 1}=200 \mathrm{~K}\right.$ and $\left.T_{C 2}=135 \mathrm{~K}\right)$. The first transition $\left(T_{C 1}\right)$ is well associated with the LSCO film and corresponds to the typical ferromagnetic-paramagnetic transition (FM-PM).
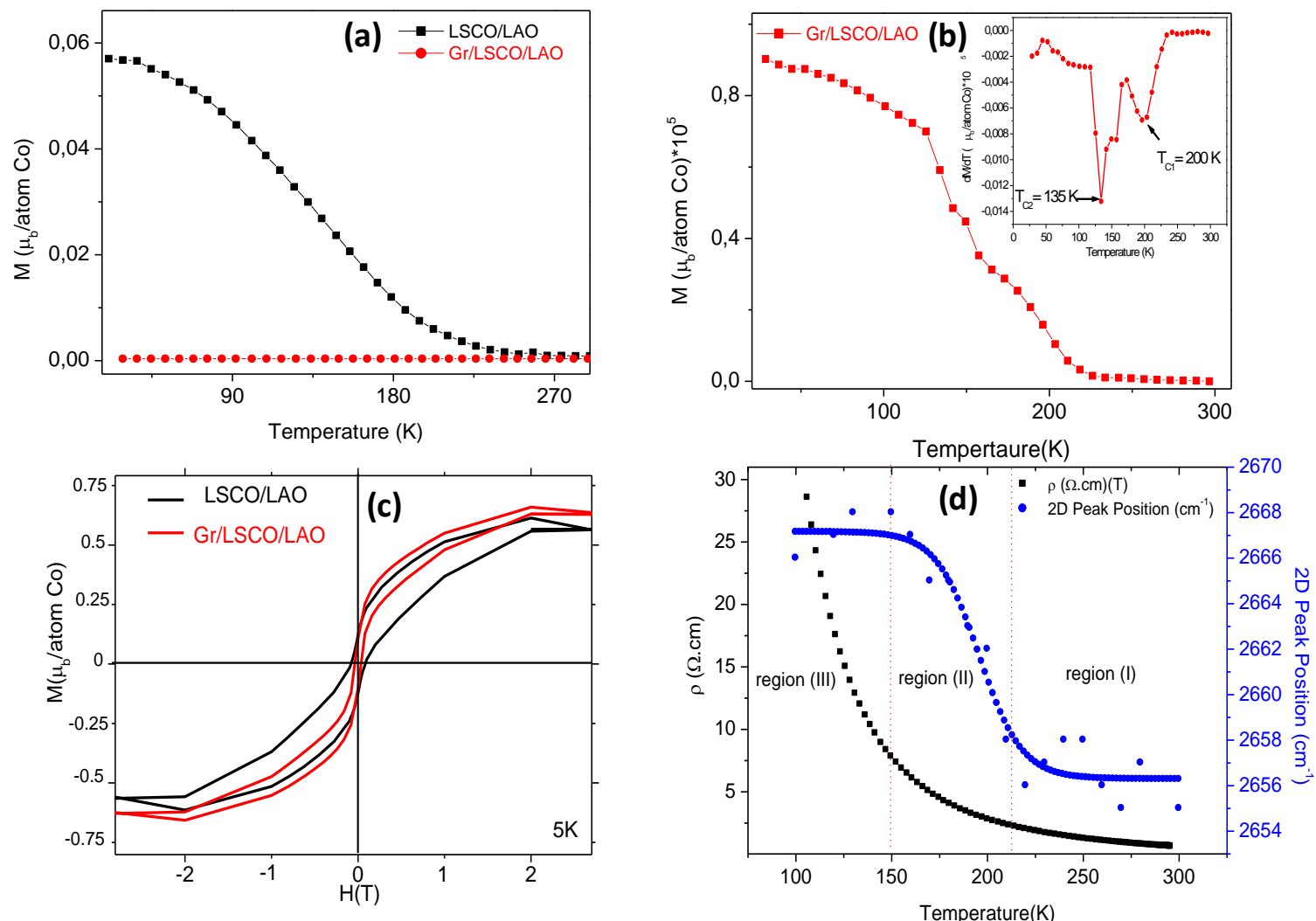

Figure 2: (Color online) (a) Temperature dependence of the magnetization (FC) (H=500 Oe) of an LSCO film grown on LAO substrate before (black) and after (red) the graphene transfer. (b) Temperature dependence of the magnetization (under FC) (H=500 Oe) of Gr/LSCO/LAO. Inset of (b) shows the $d M / d T$ used to determine the $T_{C}$ 's. (c) Hysteresis loops measured at $5 \mathrm{~K}$ before (black) and after (red) graphene transfer. (d) Temperature dependence of resistivity for the LSCO film grown on LAO substrate (black), and the temperature dependence of the 2D band frequency of G/LSCO/LAO (blue).

The second transition $\left(T_{C 2}\right)$ corresponds to an additional magnetic structure (spin and valence) of Co ions in the LSCO film induced by the graphene and likely may correspond to the uppermost part of the LSCO film in contact with the graphene layer. Fig. 2 (c) shows the 
$\mathrm{M}(\mathrm{H})$ measurements performed at $5 \mathrm{~K}$ before and after the transfer of graphene. The variations of the different parameters i.e., the saturation magnetization $\left(\mathrm{M}_{\mathrm{S}}\right)$, the remanent magnetization $\left(\mathrm{M}_{\mathrm{r}}\right)$ and the coercive field $\left(\mathrm{H}_{\mathrm{C}}\right)$ of the magnetic hysteresis curves $\mathrm{M}(\mathrm{H})$ before and after the transfer of graphene are summarized in Table 1.

\begin{tabular}{|c|c|c|c|}
\hline & $\mathrm{M}_{\mathrm{s}}\left(\mu_{\mathrm{b}} /\right.$ atomCo $)$ & $\mathrm{M}_{\mathrm{r}}\left(\mu_{\mathrm{b}} /\right.$ atomCo $)$ & $\mathrm{H}_{\mathrm{C}}(\mathrm{T})$ \\
\hline LSCO/LAO & 0.61 & 0.12 & 0.095 \\
\hline Gr-LSCO/LAO & 0.65 & 0.10 & 0.038 \\
\hline
\end{tabular}

Table 1: Magnetic saturation $\left(M_{s}\right)$, Magnetic remanence $\left(M_{r}\right)$ and coercive field $\left(H_{C}\right)$ before and after the transfer of graphene onto LSCO film.

The hysteresis curves $\mathrm{M}(\mathrm{H})$ of the $\mathrm{Gr} / \mathrm{LSCO} / \mathrm{LAO}$ seems slightly affected by the interactions with graphene. We note an increase in the value of $\mathrm{M}_{\mathrm{s}}$ and an important decrease in the value of $\mathrm{H}_{\mathrm{c}}$ when the graphene layer is transferred on the LSCO film. Therefore, these changes in both the $\mathrm{M}(\mathrm{T})$ and $\mathrm{M}(\mathrm{H})$ curves in the absence and in the presence of graphene confirm the existence of some electronic interactions between graphene and cobaltite film [32]. This resulting magnetic response can be explained by the change of the spin states in the cobaltite ions because of the electronic interactions with graphene. The shift to the low frequencies of the 2D band of the $\mathrm{Gr} / \mathrm{LSCO}$ compared to that one of $\mathrm{Gr} / \mathrm{SiO}_{2}$ we evidenced using Raman spectroscopy proves the n-type doping of the graphene [29] and consequently a depletion of electrons in the LSCO which in turn changes the Co electronic configuration and its magnetic response.
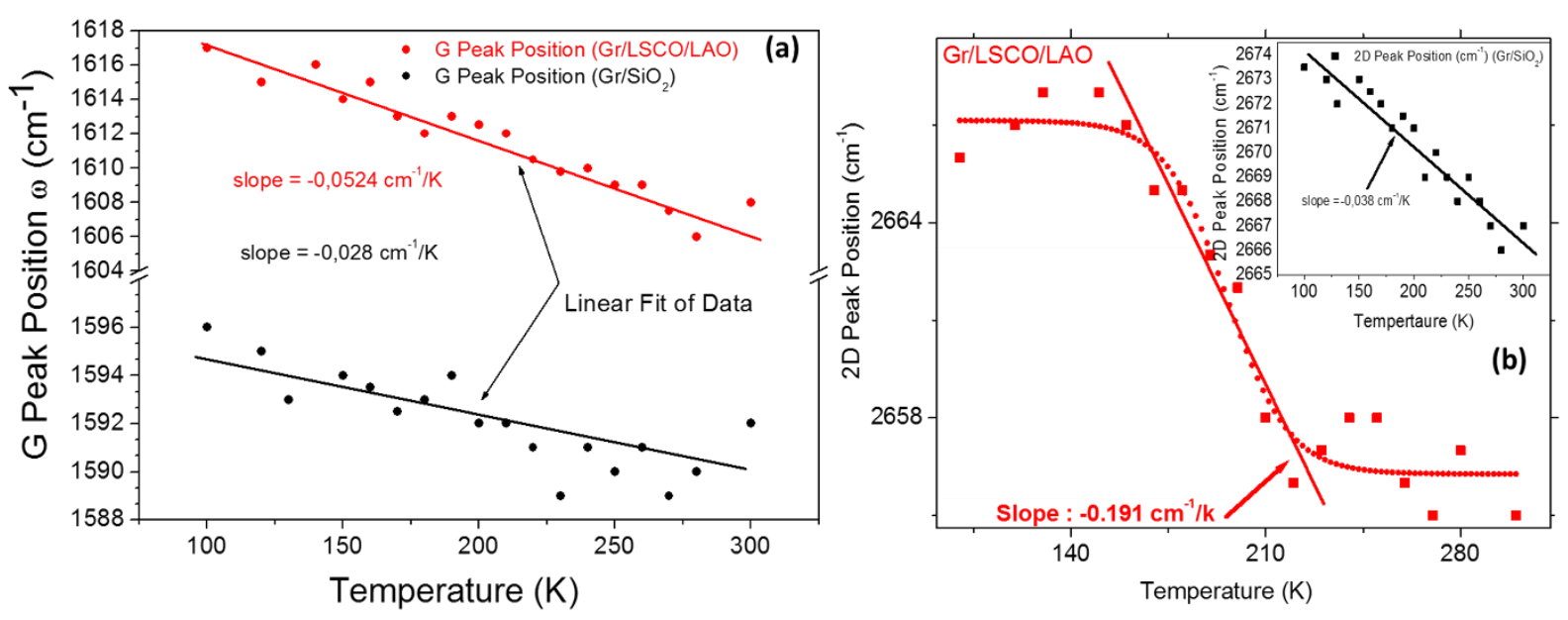

Figure 3: (Color online) (a): Temperature dependence of the $\mathrm{G}$ band frequency of $\mathrm{G} / \mathrm{SiO} 2$ (black) and G/LSCO/LAO (red). (b): Temperature dependence of the 2D band frequency of $\mathrm{Gr} / \mathrm{LSCO} / \mathrm{LAO}$, and in inset of $\mathrm{Gr} / \mathrm{SiO}_{2}$. 
The electrical and magnetic properties of the LSCO films are known to be strongly dependent on temperature [12-14]. Hence, we have performed Raman experiments as a function of temperature at the same experimental conditions for the Gr/LSCO/LAO and $\mathrm{Gr} / \mathrm{SiO}_{2}$ heterostructures. The temperature dependence of the $\mathrm{G}$ peak positions measured in the range from 100 to $300 \mathrm{~K}$ for both systems is shown in Fig 3 (a). A linear fit of the experimental data allows to take into account the temperature dependence for both systems. The slope $\partial \omega_{\mathrm{G}} / \partial \mathrm{T}$ of the evolution of the $\mathrm{G}$ band frequency versus the temperature for $\mathrm{Gr} / \mathrm{LSCO} / \mathrm{LAO}$ and $\mathrm{Gr} / \mathrm{SiO}_{2}$ is $-0.0524 \mathrm{~cm}^{-1} / \mathrm{K}$ and $-0.028 \mathrm{~cm}^{-1} / \mathrm{K}$, respectively. The almost twice higher slope for $\mathrm{Gr} / \mathrm{LSCO} / \mathrm{LAO}$ with respect to that of $\mathrm{Gr} / \mathrm{SiO}_{2}$ indicates that the electronic interaction between the graphene and the LSCO film is different from that between graphene and the $\mathrm{SiO}_{2}$ substrate. In addition, we note the lack of any specific change in the evolution of the $\mathrm{G}$ band around $T_{C}$ of the cobaltite film. Figure 3(b) shows the temperature dependence of the 2D peak position measured in the temperature range from $100 \mathrm{~K}$ to $300 \mathrm{~K}$ for $\mathrm{Gr} / \mathrm{LSCO} / \mathrm{LAO}$. A linear approximation of the data of the frequency of the $2 \mathrm{D}$ band of the $\mathrm{Gr} / \mathrm{SiO} 2$ vs. the temperature gives a slope of $-0.038 \mathrm{~cm}^{-1} / \mathrm{K}$ as depicted in the inset of Fig. 3 (b). This value of the slope is comparable to a previous report on a single-layer graphene deposited on $\mathrm{SiO}_{2} / \mathrm{Si}$ substrate with a temperature coefficient of $2 \mathrm{D}$ band Raman modes equal to $\partial \omega_{2 \mathrm{D}} / \partial \mathrm{T}=-0.026 \mathrm{~cm}^{-1} / \mathrm{K}$. Concerning the $\mathrm{Gr} / \mathrm{LSCO} / \mathrm{LAO}$ system, the temperature dependence of the frequency of the 2D band exhibits a very different behavior as shown in Fig. 3 (b). Between $\sim 210 \mathrm{~K}$ and $\sim 300 \mathrm{~K}$ (region I), we note a small variation in the $2 \mathrm{D}$ peak positions as a function of temperature. In the second region (II) i.e., for temperatures between $\sim 210 \mathrm{~K}$ and $\sim 150 \mathrm{~K}$, a monotonic increase in the $2 \mathrm{D}$ peak position is observed, with a slope of the order of $-0.191 \mathrm{~cm}^{-1} / \mathrm{K}$. Below $150 \mathrm{~K}$ (region III) a weak temperature variation of the 2D frequency is again observed. The region (I) corresponding to a slight temperature variation of the frequency of the $2 \mathrm{D}$ band can be explained by a state of electrical equilibrium between graphene and the LSCO thin film through the ntype doping of the graphene. In region (II) we note that $\partial \omega_{2 \mathrm{D}}(\mathrm{Gr} / \mathrm{LSCO} / \mathrm{LAO}) / \partial \mathrm{T}$ $=5 . \partial \omega_{2} \mathrm{D}\left(\mathrm{Gr} / \mathrm{SiO}_{2}\right) / \partial \mathrm{T}$. In this temperature region II, there are three possible explanations of such a temperature behavior of the 2D band in the Gr/LSCO system: (i) there is a structural transition in the LSCO film that can affect the graphene in-plane lattice parameters, (ii) the magnetic transition in LSCO may disturb the structural properties of the graphene and (iii) the evolution of the resistivity of the LSCO as a function of temperature may change the electronphonon coupling in graphene.

The comparison between the slopes of the two modes $\mathrm{G}$ and $2 \mathrm{D}$ show that the slopes are not equal. Indeed, in the Gr/LSCO/LAO, $\partial \omega_{2 \mathrm{D}} / \partial \mathrm{T}=3.8 \partial \omega_{\mathrm{G}} / \partial \mathrm{T}$ while $\partial \omega_{2 \mathrm{D}} / \partial \mathrm{T}=1.3 \partial \omega_{\mathrm{G}} / \partial \mathrm{T}$ in 
$\mathrm{Gr} / \mathrm{SiO}_{2}$. In the $\mathrm{Gr} / \mathrm{LSCO} / \mathrm{LAO}$ heterostructure, the changes in the 2D mode frequency are more influenced by the temperature than the G mode, which confirms the electronic origin of this variation $[29,32]$. It is clear that the electronic interaction between the Gr and the LSCO which behaves like a semiconductor is more pronounced compared to the one between $\mathrm{Gr}$ and $\mathrm{SiO}_{2}$ which in contrast exhibits an insulating behavior. We can likely attribute the evolution of the 2D band in this region (II) to a transfer of electron from graphene to LSCO films which makes the graphene becoming p-type doped. In other words, while in region I, the Fermi level in graphene is located in the conduction band (i.e., n-type semiconducting), in region II, it moves down in energy and crosses the Dirac point to reach the valence band, rendering the graphene semiconductor of p-type. Concomitantly, LSCO receives electrons from graphene when going from region I to region II which affect the Co electronic and spin state configurations. Finally, in region (III) at lower temperature, a new state of electric equilibrium is reached characterized by a low temperature evolution of the frequency of the $2 \mathrm{D}$ band.
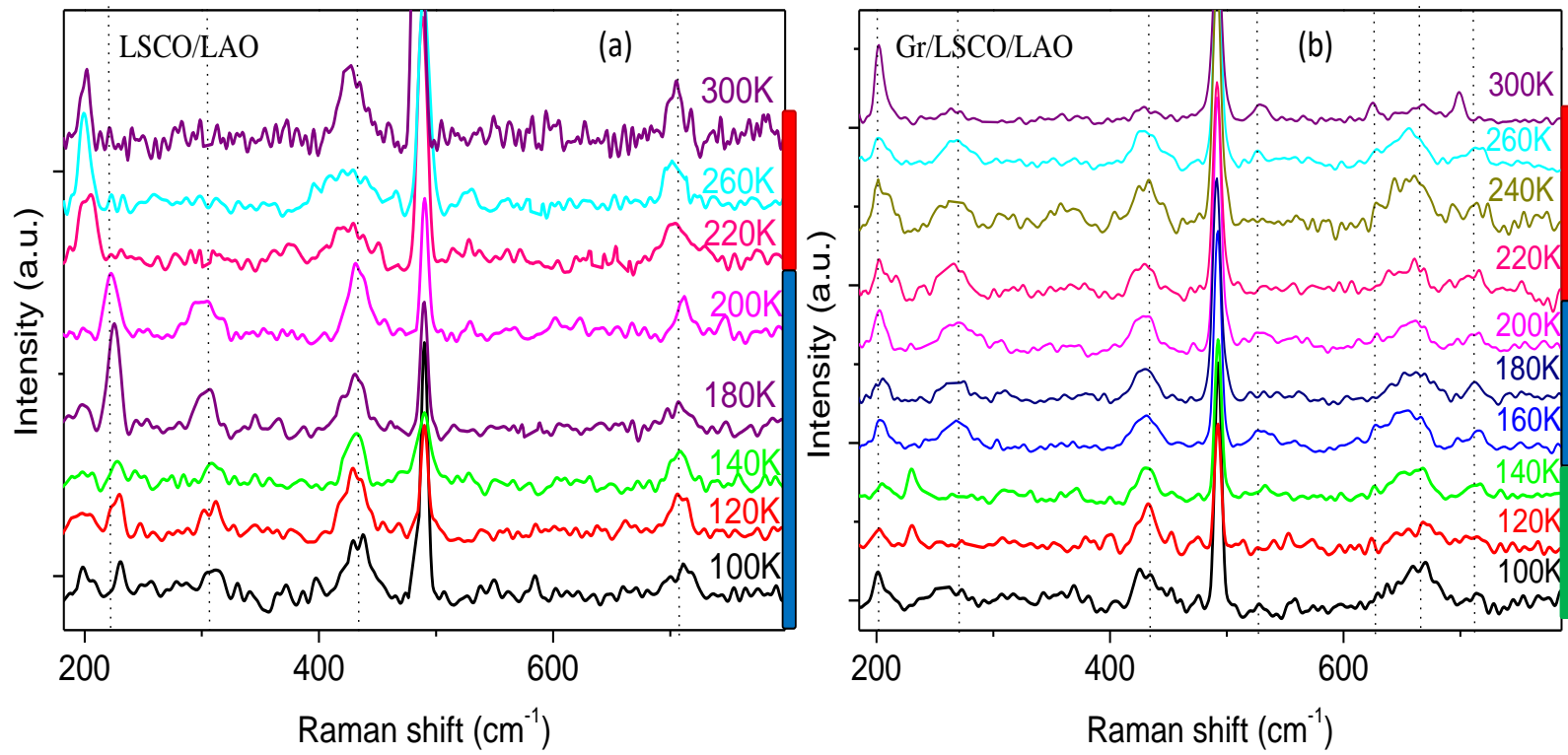

Figure 4: (Color online): Temperature dependence of Raman for LSCO/LAO film (a) before and (b) after the transfer of graphene.

To check how the electronic interactions with the graphene can affect the vibrational properties of the LSCO thin films, we have performed Raman spectra measurements as a function of temperature before and after the transfer of graphene (Fig. 4). The data were recorded between $100 \mathrm{~K}$ and $300 \mathrm{~K}$. Before the transfer of graphene (Fig. 4 (a)), the temperature 
dependence of the Raman modes shows a vibrational transition occurring below $220 \mathrm{~K}$ in the bare LSCO film with a clear shift of the $200 \mathrm{~cm}^{-1}$ mode to $220 \mathrm{~cm}^{-1}$ and the appearance of a new mode at about $300 \mathrm{~cm}^{-1}$. This transition correlates very well with the magnetic transition we evidenced in this LSCO film. The concomitance of these features indicates a substantial spin-lattice interaction in the LSCO system. After the transfer of graphene (Fig. 4 (b)) we definitely observe changes at lower temperature around $140 \mathrm{~K}$ with the disappearance of the $\sim 260 \mathrm{~cm}^{-1}$ mode and the shift of the $200 \mathrm{~cm}^{-1}$ mode to $220 \mathrm{~cm}^{-1}$, even though both modes seem to coexist. It is remarkable that these latter changes attesting of a vibrational transition in the LSCO thin film in Gr/LSCO at $140 \mathrm{~K}$ correlate very well with Region (III) we identified in the evolution of the 2D band of the graphene in Gr/LSCO. Note that the Raman spectra below 220 $\mathrm{K}$ in the bare LSCO and those below $140 \mathrm{~K}$ in Gr/LSCO are different meaning that the vibrational ground states of LSCO is obviously affected by the presence of the graphene top layer. Moreover, while the evolution of the 2D graphene band in Gr/LSCO does shows a change of behavior at $\sim 210 \mathrm{~K}$ (also associated to magnetic transition), there is no clear modification in the Raman spectra of LSCO (in Gr/LSCO system). It does not mean there are no changes, but we are not able to capture them probably because of the low resolution of our Raman spectra and/or possible tiny changes (mode broadening and/or weak intensity). Interestingly, the frequency of the $200 \mathrm{~cm}^{-1}$ mode was proposed to mainly correlate with the angle associated with the $R-3 c$ rhombohedral distortion [33] and its position can be therefore used to probe the degree of distortion as well as the critical temperature as one would expect from a soft mode. At room temperature, the intensity of the $200 \mathrm{~cm}^{-1}$ mode varies strongly after deposition and correlates with the appearance of the modes at 259, 423, 521, and $618 \mathrm{~cm}^{-1}$ which are associated to the JT distortions. This could suggest that the electronic interaction with the graphene alters LSCO rhombohedral angle by affecting the oxygen octahedra through the JT distortions. This modification could be just at the local scale however the shift towards higher frequency (i.e., $220 \mathrm{~cm}^{-1}$ ) may indicate a more profound change and a transition at long range order. This shift is observed at $210 \mathrm{~K}$ in bare LSCO while it occurs at $140 \mathrm{~K}$ in Gr/LSCO. In somehow graphene prevents the phase transition and pushes it down to lower temperature. This interpretation holds if one considers the LSCO film in Gr/LSCO made of two regions, one (the upper and closer to graphene one) overcoming the presence of graphene and another one which is too deep to "see" the presence of graphene. Nevertheless, at this stage we cannot exclude other mechanisms and further works are needed to better understand how the graphene affects the LSCO structure at short and long range and how deep the graphene top-layer interacts with the underneath LSCO film. It is also worth mentioning that a pseudo-Jahn Teller effect [34, 35] could also be involved 
here through vibronic coupling between the ground state of LSCO and its excited states due to presence of the graphene layer which may lead to lattice distortions and potentially to some ferroelectric instabilities rendering LSCO multiferroic. More works are here as well required in order to assess such possibility.

\section{Conclusion}

Based on the present experimental work studying the effects of the deposition of graphene monolayers onto LSCO films, where Raman spectra are correlated with electronic and magnetic data, we conclude that (i) Graphene is n-type doped on the LSCO around room temperature and gives it electron to LSCO on cooling; this electron depletion in graphene probably leads to a rather p-type graphene at low temperature. (ii) Due to the extra electrons provided by the graphene top-layer, LSCO which properties are very sensitive to the charge doping exhibit profound modifications of its electronic, magnetic and structural (through spin-phonon coupling) properties. As an illustration, the magnetic transition which takes place at $210 \mathrm{~K}$ in bare LSCO films is pushed down to $140 \mathrm{~K}$ with the graphene layer. The observed phenomena indicate that graphene may serve as "charge reservoir" in mixed spin-states systems like LSCO and open new perspectives for tuning spin and charge-based properties in hybrid nanostructures made of graphene and magnetic oxides.

\section{Acknowledgements}

Z.O., D.C.L and B.D. acknowledge the project "RelaxSolaire" ANR-18-CE92-0025. 


\section{References}

[1] Y. Tang, Y. Sun, Z. Cheng, Exchange bias associated with phase separation in the perovskite cobaltite $\$\{\backslash \text { mathrm }\{\mathrm{La}\}\}_{-}\{1$ lensuremath $\{-\} \mathrm{x}\}\{\backslash \operatorname{mathrm}\{\mathrm{Sr}\}\}_{-}\{\mathrm{x}\}\{\backslash \operatorname{mathrm}\{\mathrm{CoO}\}\}_{-}\{3\} \$$, Phys. Rev. B. 73 (2006) 174419. https://doi.org/10.1103/PhysRevB.73.174419.

[2] D. Fuchs, P. Schweiss, P. Adelmann, T. Schwarz, R. Schneider, Ferromagnetic order in the electron-doped system $\$\{\backslash \text { mathrm }\{\mathrm{La}\}\}_{-}\{1$ lensuremath $\{-$

\} $\mathrm{x}\}\{\backslash \operatorname{mathrm}\{\mathrm{Ce}\}\}_{-}\{\mathrm{x}\} \backslash$ mathrm $\{\mathrm{Co}\}\{\backslash \text { mathrm }\{\mathrm{O}\}\}_{-}\{3\} \$$, Phys. Rev. B. 72 (2005) 014466. https://doi.org/10.1103/PhysRevB.72.014466.

[3] D. Phelan, D. Louca, K. Kamazawa, M.F. Hundley, K. Yamada, Influence of the ionic size on the evolution of local Jahn-Teller distortions in cobaltites, Phys. Rev. B. 76 (2007) 104111. https://doi.org/10.1103/PhysRevB.76.104111.

[4] J.W. Freeland, J.X. Ma, J. Shi, Ferromagnetic spin-correlations in strained $\mathrm{LaCoO} 3$ thin films, Appl. Phys. Lett. 93 (2008) 212501. https://doi.org/10.1063/1.3027063.

[5] J. Androulakis, P. Migiakis, J. Giapintzakis, La0.95Sr0.05CoO3: An efficient room-temperature thermoelectric oxide, Appl. Phys. Lett. 84 (2004) 1099-1101. https://doi.org/10.1063/1.1647686.

[6] M.A. Torija, M. Sharma, J. Gazquez, M. Varela, C. He, J. Schmitt, J.A. Borchers, M. Laver, S. El-Khatib, C. Leighton, Chemically Driven Nanoscopic Magnetic Phase Separation at the SrTiO3(001)/La1-xSrxCoO3 Interface, Advanced Materials. 23 (2011) 2711-2715. https://doi.org/10.1002/adma.201100417.

[7] M. Kubicek, Z. Cai, W. Ma, B. Yildiz, H. Hutter, J. Fleig, Tensile Lattice Strain Accelerates Oxygen Surface Exchange and Diffusion in La1-xSrxCoO3- $\delta$ Thin Films, ACS Nano. 7 (2013) 3276-3286. https://doi.org/10.1021/nn305987x.

[8] J. Wu, C. Leighton, Glassy ferromagnetism and magnetic phase separation in $\$\{\backslash \text { mathrm }\{\text { La }\}\}_{-}\{1$ lensuremath $\{-\} \mathrm{X}\}\{\backslash \operatorname{mathrm}\{\mathrm{Sr}\}\}_{-}\{\mathrm{x}\}\{\backslash \operatorname{mathrm}\{\mathrm{CoO}\}\}_{-}\{3\} \$$, Phys. Rev. B. 67 (2003) 174408. https://doi.org/10.1103/PhysRevB.67.174408.

[9] D.P. Kozlenko, N.O. Golosova, Z. Jirák, L.S. Dubrovinsky, B.N. Savenko, M.G. Tucker, Y. Le Godec, V.P. Glazkov, Temperature- and pressure-driven spin-state transitions in $\$\{\text { Imathrm }\{\mathrm{LaCoO}\}\}_{-}\{3\} \$$, Phys. Rev. B. 75 (2007) 064422. https://doi.org/10.1103/PhysRevB.75.064422.

[10]A. Podlesnyak, S. Streule, J. Mesot, M. Medarde, E. Pomjakushina, K. Conder, A. Tanaka, M.W. Haverkort, D.I. Khomskii, Spin-State Transition in $\$\{\text { mathrm }\{\mathrm{LaCoO}\}\}_{-}\{3\} \$$ : Direct Neutron Spectroscopic Evidence of Excited Magnetic States, Phys. Rev. Lett. 97 (2006) 247208. https://doi.org/10.1103/PhysRevLett.97.247208.

[11]F. Guillou, Q. Zhang, Z. Hu, C.Y. Kuo, Y.Y. Chin, H.J. Lin, C.T. Chen, A. Tanaka, L.H. Tjeng, V. Hardy, Coupled valence and spin state transition in $\left(\operatorname{Pr} \$\{\}_{-}\{0.7\} \$ \operatorname{Sm} \$\{\}_{-}\{0.3\} \$\right) \$\{\}_{-}\{0.7\} \$ C a d\{\}_{-}\{0.3\} \$ \operatorname{CoO} \$\{\}_{-}\{3\} \$$, Phys. Rev. B. 87 (2013) 115114. https://doi.org/10.1103/PhysRevB.87.115114.

[12]T. Das, T. Saha-Dasgupta, Spin-state transition in unstrained \& strained ultra-thin BiCoO3 films, Dalton Trans. 44 (2015) 10882-10887. https://doi.org/10.1039/C5DT00025D.

[13]D. Phelan, D. Louca, K. Kamazawa, S.-H. Lee, S.N. Ancona, S. Rosenkranz, Y. Motome, M.F. Hundley, J.F. Mitchell, Y. Moritomo, Spin Incommensurability and Two Phase Competition in Cobaltites, Phys. Rev. Lett. 97 (2006) 235501. https://doi.org/10.1103/PhysRevLett.97.235501.

[14]Z. Othmen, A. Schulman, K. Daoudi, M. Boudard, C. Acha, H. Roussel, M. Oueslati, T. Tsuchiya, Structural, electrical and magnetic properties of epitaxial $\mathrm{La} 0.7 \mathrm{Sr} 0.3 \mathrm{CoO} 3$ thin films grown on SrTiO3 and LaAlO3 substrates, Applied Surface Science. 306 (2014) 60-65. https://doi.org/10.1016/j.apsusc.2014.03.034.

[15]A.K. Geim, K.S. Novoselov, The rise of graphene, Nature Materials. 6 (2007) 183-191. https://doi.org/10.1038/nmat1849.

[16]A.S. Mayorov, R.V. Gorbachev, S.V. Morozov, L. Britnell, R. Jalil, L.A. Ponomarenko, P. Blake, K.S. Novoselov, K. Watanabe, T. Taniguchi, A.K. Geim, Micrometer-Scale Ballistic Transport in Encapsulated Graphene at Room Temperature, Nano Lett. 11 (2011) 2396-2399. https://doi.org/10.1021/nl200758b.

[17]J. Hicks, A. Tejeda, A. Taleb-Ibrahimi, M.S. Nevius, F. Wang, K. Shepperd, J. Palmer, F. Bertran, P. Le Fèvre, J. Kunc, W.A. de Heer, C. Berger, E.H. Conrad, A wide-bandgap metal- 
semiconductor-metal nanostructure made entirely from graphene, Nature Physics. 9 (2013) 49 54. https://doi.org/10.1038/nphys2487.

[18]J.W. Suk, A. Kitt, C.W. Magnuson, Y. Hao, S. Ahmed, J. An, A.K. Swan, B.B. Goldberg, R.S. Ruoff, Transfer of CVD-Grown Monolayer Graphene onto Arbitrary Substrates, ACS Nano. 5 (2011) 6916-6924. https://doi.org/10.1021/nn201207c.

[19]D. Geng, H. Wang, G. Yu, Graphene Single Crystals: Size and Morphology Engineering, Advanced Materials. 27 (2015) 2821-2837. https://doi.org/10.1002/adma.201405887.

[20]M. Weser, E.N. Voloshina, K. Horn, Y.S. Dedkov, Electronic structure and magnetic properties of the graphene/Fe/Ni(111) intercalation-like system, Phys. Chem. Chem. Phys. 13 (2011) 75347539. https://doi.org/10.1039/C1CP00014D.

[21]R. Decker, J. Brede, N. Atodiresei, V. Caciuc, S. Blügel, R. Wiesendanger, Atomic-scale magnetism of cobalt-intercalated graphene, Phys. Rev. B. 87 (2013) 041403. https://doi.org/10.1103/PhysRevB.87.041403.

[22]D. Li, H. Zhao, L. Li, B. Mao, M. Chen, H. Shen, W. Shi, D. Jiang, Y. Lei, Graphene-Sensitized Perovskite Oxide Monolayer Nanosheets for Efficient Photocatalytic Reaction, Advanced Functional Materials. 28 (2018) 1806284. https://doi.org/10.1002/adfm.201806284.

[23]J.-B. Wu, M.-L. Lin, X. Cong, H.-N. Liu, P.-H. Tan, Raman spectroscopy of graphene-based materials and its applications in related devices, Chem. Soc. Rev. 47 (2018) 1822-1873. https://doi.org/10.1039/C6CS00915H.

[24]J.M. Caridad, F. Rossella, V. Bellani, M. Maicas, M. Patrini, E. Díez, Effects of particle contamination and substrate interaction on the Raman response of unintentionally doped graphene, Journal of Applied Physics. 108 (2010) 084321. https://doi.org/10.1063/1.3500295.

[25]K. Daoudi, T. Tsuchiya, T. Nakajima, A. Fouzri, M. Oueslati, Epitaxial growth of $\mathrm{La} 0.7 \mathrm{Sr} 0.3 \mathrm{CoO} 3$ thin films on $\mathrm{SrTiO} 3$ substrates by metal-organic deposition, Journal of Alloys and Compounds. 506 (2010) 483-487. https://doi.org/10.1016/j.jallcom.2010.07.035.

[26]H. Ajlani, R. Othmen, M. Oueslati, A. Cavanna, A. Madouri, Graphene-capped InAs/GaAs quantum dots, Journal of Vacuum Science \& Technology B. 31 (2013) 020602. https://doi.org/10.1116/1.4790365.

[27]R. Othmen, K. Rezgui, A. Cavanna, H. Arezki, F. Gunes, H. Ajlani, A. Madouri, M. Oueslati, Improvement of the quality of graphene-capped InAs/GaAs quantum dots, Journal of Applied Physics. 115 (2014) 214309. https://doi.org/10.1063/1.4880338.

[28]R. Othmen, H. Arezki, H. Ajlani, A. Cavanna, M. Boutchich, M. Oueslati, A. Madouri, Direct transfer and Raman characterization of twisted graphene bilayer, Appl. Phys. Lett. 106 (2015) 103107. https://doi.org/10.1063/1.4914309.

[29]A. Das, S. Pisana, B. Chakraborty, S. Piscanec, S.K. Saha, U.V. Waghmare, K.S. Novoselov, H.R. Krishnamurthy, A.K. Geim, A.C. Ferrari, A.K. Sood, Monitoring dopants by Raman scattering in an electrochemically top-gated graphene transistor, Nature Nanotechnology. 3 (2008) 210-215. https://doi.org/10.1038/nnano.2008.67.

[30]A. Ishikawa, J. Nohara, S. Sugai, Raman Study of the Orbital-Phonon Coupling in $\$\{\backslash \text { mathrm }\{\mathrm{L}\} \backslash \text { mathrm }\{\mathrm{a}\} \backslash \mathrm{mathrm}\{\mathrm{C}\} \backslash \mathrm{mathrm}\{\mathrm{o}\} \backslash \operatorname{mathrm}\{\mathrm{O}\}\}_{-}\{3\}$, Phys. Rev. Lett. 93 (2004) 136401. https://doi.org/10.1103/PhysRevLett.93.136401.

[31]Z. Othmen, O. Copie, K. Daoudi, M. Boudard, P. Gemeiner, M. Oueslati, B. Dkhil, Spin transitions in $\mathrm{La} 0.7 \mathrm{Ba} 0.3 \mathrm{CoO} 3$ thin films revealed by combining Raman spectroscopy and X-ray diffraction, Journal of Applied Physics. 120 (2016) 015308. https://doi.org/10.1063/1.4955220.

[32]D. Yoon, Y.-W. Son, H. Cheong, Negative Thermal Expansion Coefficient of Graphene Measured by Raman Spectroscopy, Nano Lett. 11 (2011) 3227-3231. https://doi.org/10.1021/nl201488g.

[33]M.V. Abrashev, A.P. Litvinchuk, M.N. Iliev, R.L. Meng, V.N. Popov, V.G. Ivanov, R.A. Chakalov, C. Thomsen, Comparative study of optical phonons in the rhombohedrally distorted perovskites $\mathrm{LaAlO}_{3}$ and $\mathrm{LaMnO}_{3}$, Phys. Rev. B. 59 (1999) 4146-4153. https://doi.org/10.1103/PhysRevB.59.4146.

[34] I. B. Bersuker, Phys. Rev. Lett., 108, 137202 (2012)

[35] L. Weston, X. Y. Cui, S. P. Ringer, and C. Stampfl, Phys. Rev. B, 93, 165210 (2016) 